\title{
Butterfly Voices - \\ Creative Self-Representation of Migrant Sex Workers
}

\author{
Alvis Choi \& Elene Lam
}

\begin{abstract}
Butterfly is an Asian and Migrant Sex Workers Support Network based in Toronto, Ontario, Canada. The organization was founded upon the belief that migrant sex workers, like all workers, are entitled to respect and human rights. The following essay on Butterfly Voices was written by the project's co-creators, Alvis Choi and Elene Lam, and details the creative energies, political vision, and methodological steps that went into bringing the project to life.
\end{abstract}

\section{Keywords}

migrant sex workers, art-based projects, self-representation 


\section{Butterfly Voices - \\ Creative Self-Representation of Migrant Sex Workers}

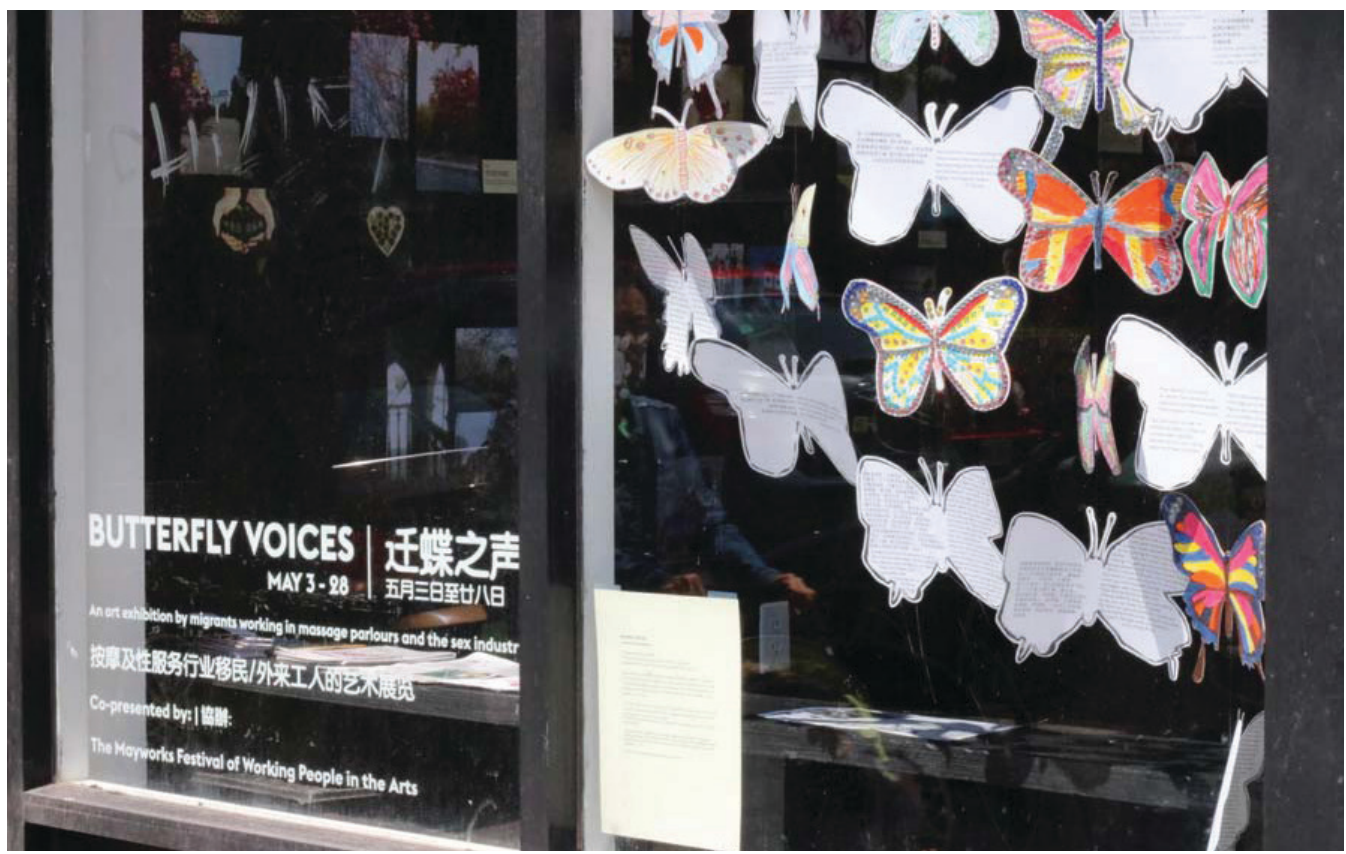

\section{Whispering Wishes}

Butterfly Voices is an ongoing arts-based project that seeks to create opportunities for the self-representation of Asian and migrant sex workers in Toronto/Three Fire Territories and beyond. The project began in early 2015 in light of the negative stereotypes and stigma associated with migrant sex workers. We invited self-identified migrant sex workers around the world, many of whom are silenced because of the lack of understanding and systemic oppression around sex work, to participate in Butterfly Voices and share their stories.

One of the popular myths circulated by the state and the media about migrant sex workers is that these workers are always victims of human or sex trafficking; that they are only involved in sex work because they are forced to do so. In reality, migrant sex workers often have varying levels of agency in different aspects of their lives. Their immigration status' and identities also vary. The notion that migrant sex workers are always victims inaccurately portrays them as inherently weak and naïve, as opposed to agentic human beings who make life choices and compromises just like everyone else, and subsequently diverts public attention away from the systemic forces of oppression that put migrant sex workers' lives at risk on a daily basis. These forces of oppression include, but are not limited to, the criminalization and legalization of sex work, white heteropatriarchy, and whorephobia (Durisin, Meulen \& Bruckert 2018; Gallant et. al 2018).

Butterfly Voices uses art and storytelling to engage migrant sex workers in Toronto/Three Fire Territories and beyond by exploring self-determination through creative expression, thereby amplifying the voices of these workers. The first iteration of Butterfly Voices was presented at the Migrant Sex Workers' Justice Forum, held on May 12th, 2015 at Ryerson University in Toronto, Ontario, as an installation (Choi \& Lam, 2016). We distributed black and white copies of pencil-outlined butterflies drawn by Montreal-based artist Sarah Mangle at migrant sex workers' workplaces in Toronto (including massage parlours and private workplaces) and asked participants to respond to three questions. Their answers were submitted with the paper butterflies that they coloured and decorated. 


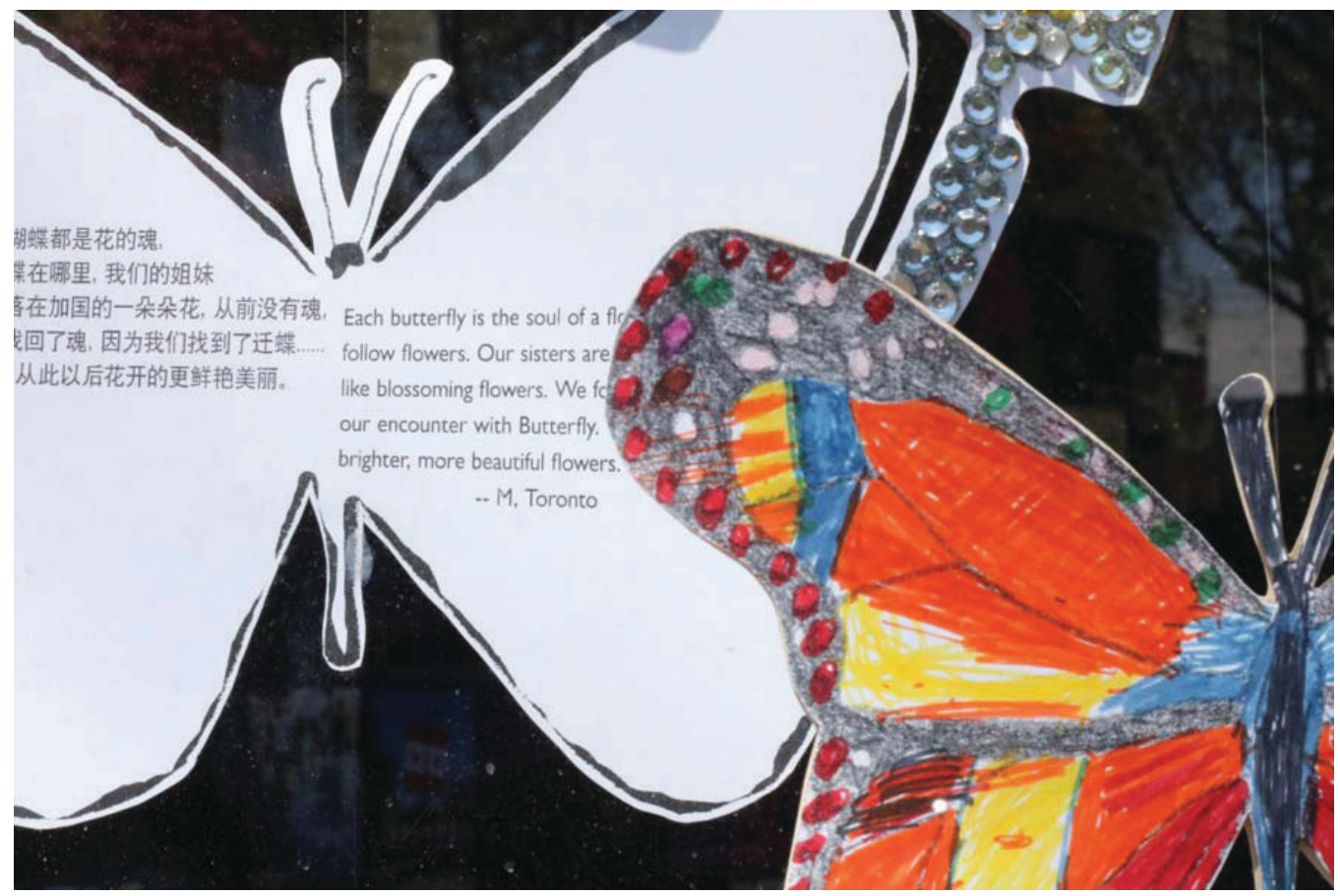

We used butterflies, for which migration is a given, as a metaphor and a starting point. We invited participants to answer the following questions:

1. What wishes would you whisper to a butterfly if it could make them come true?

2. What is empowering about being a migrant sex worker? How does sex work help you achieve your dream?

3. How do migrant sex workers contribute to society, their clients, and your community?

The responses that we received reflect a wide range of ideologies, concerns, and politics amongst migrant sex workers. We received over 40 submissions - the majority of which were from Toronto, with some from Montreal, Hong Kong, Berlin, Scotland, and France.

\section{When a community artist meets a community activist...}

In this essay we share the story that motivated us to launch Butterfly Voices, the methods that we used when working with migrant sex workers, the outcome of these methods, and our overall vision for the project. From an organizer's point of view, Butterfly Voices has been a delightful and meaningful collaboration between a community artist and a grassroots activist. Both of us believe in the effectiveness of art and creativity in community organizing and the liberatory and transformative potential that it brings. As a community artist, performer, and facilitator, Alvis Choi has conceived a number of social practice-based projects and performances that engage the Chinese-speaking community, the queer of colour community, and a larger art audience. Their work often addresses themes of self-representation, truth, and empowerment within a framework that counters dominant white heteronormative ideologies. As a grassroots activist, Elene Lam has been an advocate for sex workers, migrants, and labour and genderbased justice for more than fifteen years in both Asia and Canada. Her work intersects art, activism, advocacy, education, and culture. For her, art is not only a means by which to engage marginalized communities, it is also a tool for building meaningful 


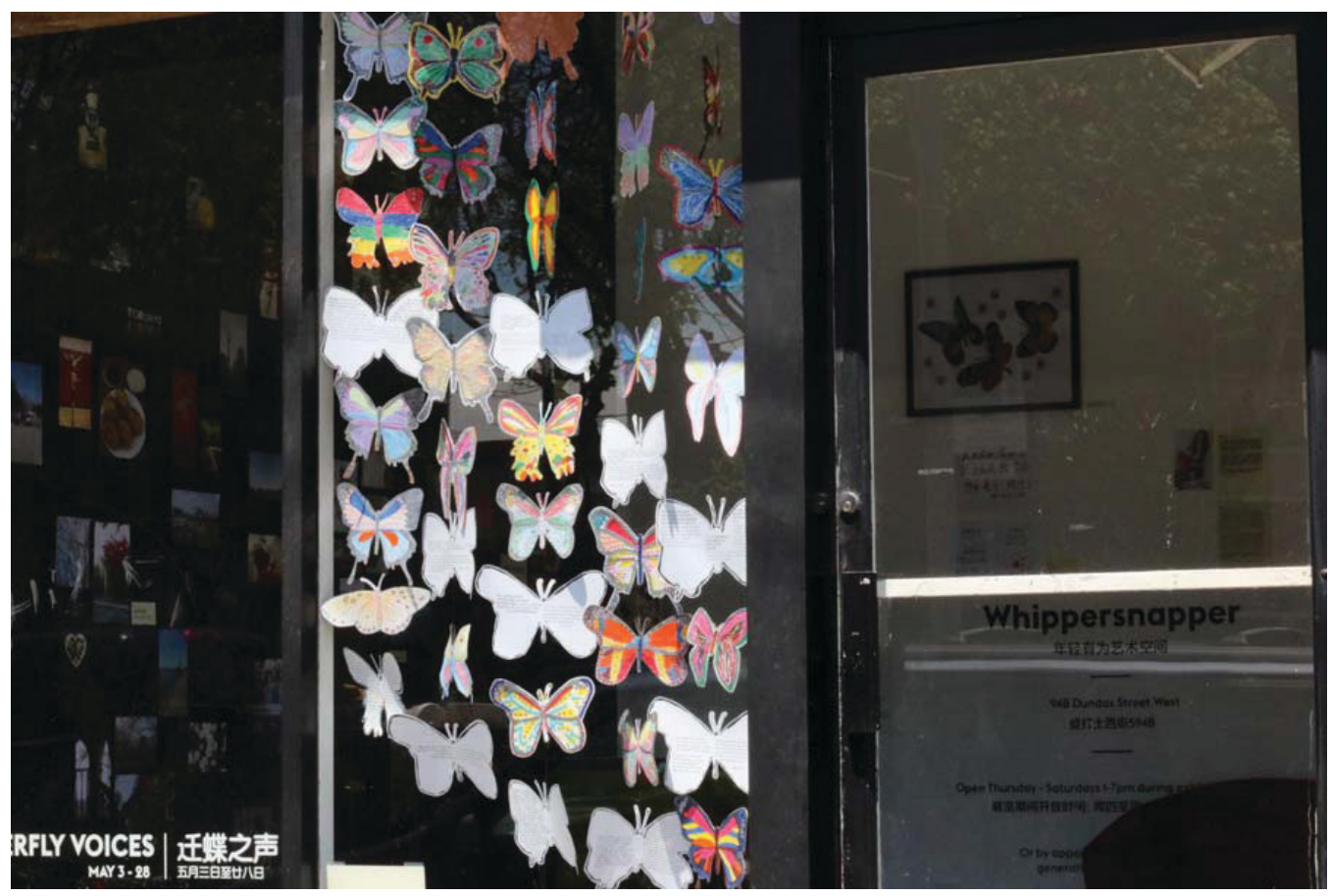

connections between diverse community members, helping to mobilize and develop leadership skills within these communities. When the two of us met, we gradually developed a trusting relationship and began to work together on several projects that would combine our skill sets.

In the mainstream sex worker movement in North America, the voices of racialized sex workers are often missing (Gallant et al., 2018; Lam \& Gallant, 2018). While issues such as race and class are inseparable from sex work, they remain underaddressed in these movements. The experiences of racialized and/or poor sex workers are seldom centered, making these movements inaccessible to individuals who are the most marginalized within the sex worker community. For migrant sex workers, an additional aspect that obstructs their participation within the mainstream sex worker community is their (im)migration status. The rhetoric of migrant sex workers as trafficked subjects creates barriers that prevent them from getting involved with or benefiting from the mainstream sex worker movement (Lam, 2016; Lepp, 2018). Through media and propaganda campaigns, migrant sex workers are purposefully posed as threats or burdens to the state, the legal system, and law enforcement (Gallant et. al 2018). They are often targeted by law enforcement through raids, harassment, and other racist and misogynist practices (Lam, 2018a; Lam, 2018b). Further, they are not protected by the mainstream movement as it is inaccessible to non-English-speaking sex workers whose experiences are unique due to their cultural background and immigration status. Lastly, these workers are also subjected to the stereotypical image of being weak, ignorant, powerless, and naïve, which has to do with the racist and misogynist perception of Asian women and women of colour as submissive (Lam \& Lepp, 2019).

The anti-state rhetoric that is commonly used within and by the mainstream sex worker movement, as a strategy, lacks consideration of the lived experiences of many marginalized sex workers. For instance, a common tactic used within this movement is to rally against police and state intervention and regulation. However, participating in a rally that gathers the oppressed and advocates on behalf of fighting against police and state authority might feel life threatening to a migrant sex worker 
who comes from mainland China, where any thought or action that consists of anti-government ideologies is deemed illegal. The particular forms in which the current mainstream sex workers movement takes place, such as rallying, petitioning, policy change advocacy, the sharing of first-hand experiences, etc., often require a high level of visibility from participating workers, thereby creating additional barriers that make it especially challenging for migrant sex workers to engage in the dialogue (Lam, 2016). Butterfly Voices aims to create a culturally sensitive platform for migrant sex workers to participate in social organizing in ways that ensure that they are safe and heard and that their comfort levels and preferences are being prioritized.

\section{Stories to Tell, Workers to Empower}

When we first conceptualized this project, we went through a series of discussions and negotiations about the methodology and ethics of portraying migrant sex workers' experiences. We asked: How do we design a process of participation in which participants feel empowered and that their experiences are accurately represented in the knowledge we co-produce? We had considered interviewing some of the local migrant sex workers - with whom Elene has existing relationships with. From these conversations, we would draft an account of the workers' experiences in the form of storytelling and bring these written stories back to the workers for them to review and revise, ensuring that they were comfortable with everything that we aimed to publish. However, we did not end up using this method as we were particularly cautious about influencing the participants' stories with our own assumptions and pre-existing narratives. We wanted to ensure that we were representing the workers' lives as accurately and as genuinely as possible, and we remained cognizant of the fact that there was always a possibility of misrepresentation - despite how conscious we were of the problems around speaking for others (Alcoff, 1991).

We were aware that we needed to design a process of participation that would be accessible to many. In this process, we learned that we had to consider the following factors for an art-based project aimed at successfully engaging the migrant sex worker community:
1. Participants' time constraints

2. Privacy and confidentiality

3. Content relevance to participants' everyday lives

4. Capacity to be fully present during participation

5. Participants' self-identification of the work that they do and the rhetoric that they use to discuss the issues of migrant sex workers' rights

Amongst the local migrant sex workers in Toronto whom we reached out to, many of them worked at a shared workspace and had unpredictable schedules. Thus, we had to design a process that allowed for some flexibility and was easy to participate in. Conventional community arts often emphasize participants coming together at a set time and place. However, in our case, this was not possible. We therefore brought materials and art supplies to the workers' workplaces and invited them to work on the paper butterflies whenever they had time between work sessions. Elene would then pick up the artwork from the workers and follow up with them regarding their responses.

We expected this to be a new experience for many of the workers - to participate in an art-based project and to tell their story to the public. It was therefore necessary for us to invest in explaining the intention of our project to them and ensuring that the workers were well informed. Elene also had many conversations with the workers to ensure that we collected their stories in a respectful way. Some of this work was done in person, while some of it was done over text messages, instant messaging, or phone calls. Participants remained anonymous as they desired. Finally, we wanted the project to be inviting, enjoyable, and intriguing for the workers Unlike sex workers who are English-speaking, relatively tech-savvy, and have the option to work from home on their own schedule with the help of the Internet, migrant sex workers tend to work long hours. We thus hoped that by bringing art into their workplaces, we could encourage migrant sex workers to tell their stories in a creative and fun way.

Through this iteration of Butterfly Voices, we learned that the invisibility and precarious situation of migrant sex workers meant that we needed to emphasize an engaged research process that was 


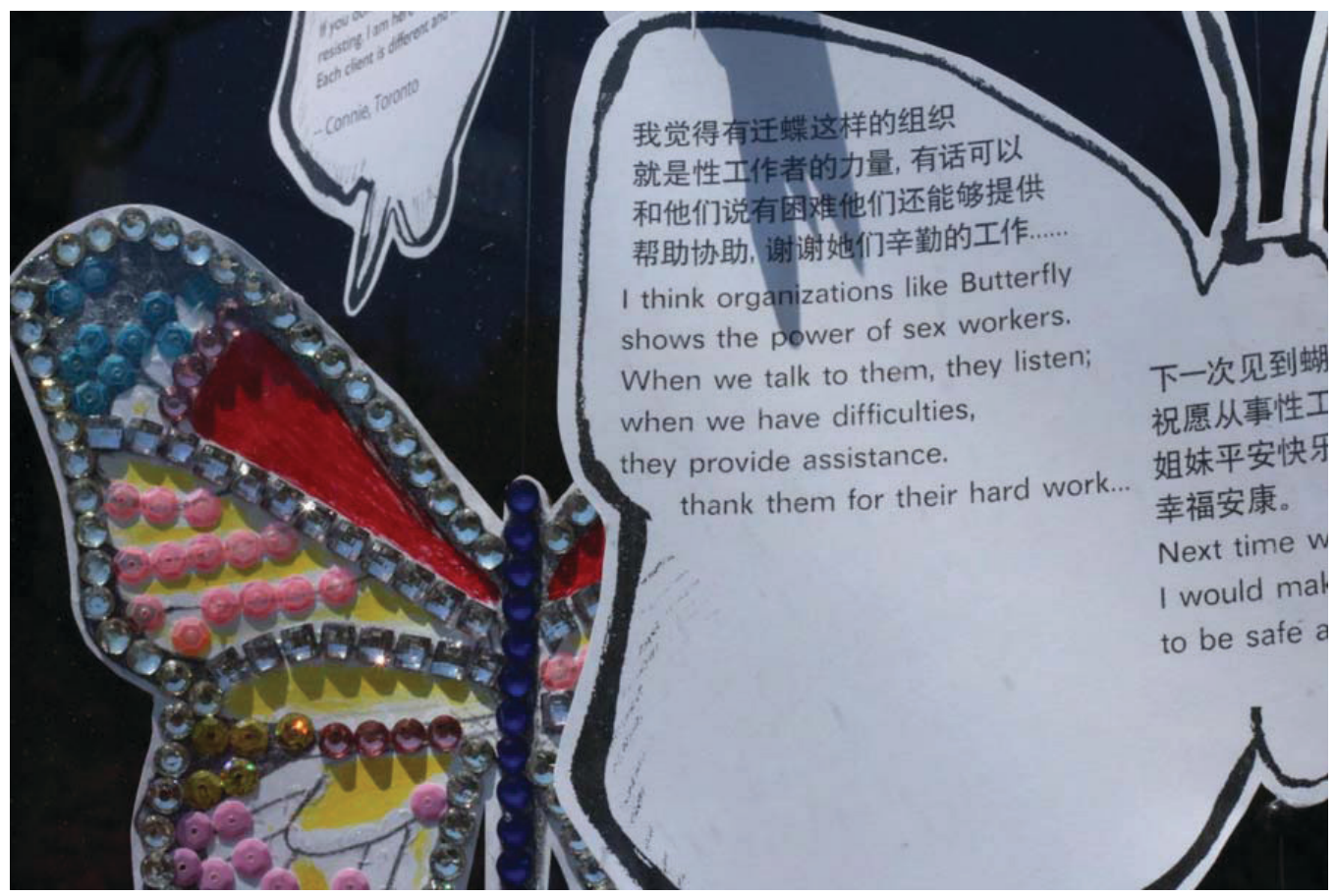

personal and custom-made. In doing so, we were able to make Butterfly Voices accessible to migrant sex workers who may or may not have a flexible schedule and thus the time and/or energy to invest in such a project. Accordingly, the method we adopted considered the conditions of the workers' everyday lives and prioritized the needs and desires of the participants. Building on existing relationships and experiences with the community, Butterfly Voices is one of the first art-based projects in Toronto that engages migrant sex workers directly in their workplaces, thereby allowing them the opportunity to not only represent themselves, but to do so on their own terms.

\section{Breathing Room for Creativity}

For migrant sex workers who do not have the privilege to be visible, as they risk being targeted by law enforcement agents on a daily basis, we wanted to avoid asking participants to directly share their experiences of and with sex work, which would only create stress for them. Instead, we used paper butterflies as a bridge to open up a space for storytelling. In particular, artist Sarah Mangle created six different butterfly drawings for participants to engage with. We offered a variety of affordable art supplies to workers to colour and decorate the black and white paper butterflies Crayons, markers, colour pencils, glitter, stickers, sequins. This provided variety. Yet, at the same time, Mangle's drawing style offered coherency to the overall presentation. In terms of artistic medium and materials, there were some limitations. We had to consider practicality - Is it relatively clean or messy to use this material? Does it require additional preparation such as water (e.g. watercolour)? Is it quick and easy for the worker to tidy up when a client shows up? We wanted to ensure that this art making experience created joy and pleasure rather than more work for the participants.

It was interesting to see how participants used the butterfly templates to express themselves, and we learned a lot about the community as a result. For one, we witnessed the dynamics among workers by observing how they shared the art supplies within the workplace. Secondly, we learned how we could do better in removing barriers to participation, offering workers options and flexibility to encourage them to participate in the project on their own terms. How they participated spoke to their work conditions, interests, and capacities; and we wanted to respect that. Further, not everyone responded to 
the questions we asked. Some of them chose to only colour or decorate the butterflies. Others responded to only one or two of our questions. One participant even made small sculptures out of the stickers we provided!

It is also important to note that there wasn't really a unifying voice of any sort. All the butterflies created were unique and different. If you read them closely enough, you can see that workers come from different places, backgrounds and cultures, and political locations. This diversity, we think, is representative of the larger community of migrant sex workers, as it highlights the heterogeneity of the many voices that exist within it.

\section{As Always, It Could Be Better}

We had originally envisioned the project to be a global one. While we received some submissions from cities other than Toronto, globalizing Butterfly Voices required a lot more than simply broadening the scope of our submissions. For one, we did not have the capacity to translate the call for submissions into languages other than English and Chinese. This is something we were criticized for, as a multilingual call for submissions would have encouraged a much wider participation from migrant sex workers from all around the world. Another issue we encountered was the choice of words we decided to use in the call. In the Chinese version, we used the term "massage parlour workers" instead of "sex workers" as this was a more common way of self-identifying amongst the Chinese workers whom we spoke to. However, we had chosen, in the English version, to go with "sex workers." But we received feedback from a worker outside of our existing network who reminded us that we should include a variety of terms that migrant sex workers of different socioeconomic classes might call themselves, such as hookers, hustlers, or prostitutes. This comment highlighted our initial lack of acknowledgement of the class differences among the heterogeneous community of migrant sex workers. Although the term "sex worker" is the politically correct word to use, it does not necessarily speak to everyone whose voice is important to the sex workers' rights movement, as some workers simply do not identify with the term "sex worker." This experience taught us that, for our project to be successful, we must constantly investigate whether our methods, and overall approach, is informed by an intersectional understanding of the systemic issues that affect the entire migrant sex workers community.

\section{Connecting without Outing}

The first iteration of Butterfly Voices was presented as an installation at the Migrant Sex Workers' Justice Forum, which was attended by over 120 participants, including sex workers, sex worker rights activists, academics, legal professionals, journalists, social workers, government officials, artists, and community members. Each butterfly in the installation was coloured and decorated by our participants. Each butterfly represented the voice of a migrant sex worker. The exhibition became a platform for migrant sex workers' experiences to be visualized without "outing" them. Through the empowering acts of first-person storytelling and art making, the exhibition offered a refreshing lens through which the public could gain a better understanding of the diverse lives of migrant sex workers. The materiality of the butterflies coming together signified a connection between migrant sex workers. At the end of the first iteration, we designed flyers and stickers that featured all of the butterflies that were thoughtfully coloured and decorated by each worker. We then distributed these swags back to the community. Many of the participants were delighted to see their own art printed on stickers and flyers. They did not expect this kind of professionally finished product and, just as we did, found pride and satisfaction in their work. They also expressed feelings of being respected and valued. The tangibility of the documentation featuring all of our submissions side by side created a sense of connection. Workers could see, not only their own art in print, but the art of other workers on the same print surface. This follow up work contributed to making the project meaningful to the participants. It also helped to build a personal relationship between them and the society, as well as strengthen the sex workers' rights movement as a whole, which often excludes the voices of marginalized groups. In this sense, the follow up work we did after the first iteration of Butterfly Voices was just as important as the actual process of creating the installation.

A critical and foundational part of our activism is to make space for migrant sex workers to speak for themselves. We recognize that each submission we 
received played a significant role in the ultimate goal of Butterfly Voices: to reduce and remove the stigma surrounding migrant sex work. Through this ongoing project, we hope to demystify sex work, especially sex work provided by migrant workers, through creative art that is grounded in lived experience. We give gratitude to each and every migrant sex worker who contributed their thoughts and stories to Butterfly Voices, as well as those who witnessed the process and outcome of our first installation. After the success of this first phase of Butterfly Voices, the team at Butterfly, in concert with our collaborators, decided to continue to empower migrant sex workers to engage in art-based conversations about their rights. To this point, Butterfly Voices has been shown at Whippersnapper Gallery and Workers Action Centre in Toronto, Ryerson University, and Central Cinema in Seattle, and the outcome has been significant. We align ourselves with the migrant sex workers movement under the strong belief that sex work is work. Butterfly Voices will continue to bring these perspectives to the foreground of social justice organizing, while laboring to improve the actual working conditions of migrant sex workers, in addition to the working conditions of other workers in the broader migrant workers' movement. We seek your support in meeting our goal to nurture Butterfly Voices into a large-scale exhibition and, eventually, a global project.

\section{References}

Alcoff, L. (1991). The problem of speaking for others. Cultural critique, (20), 5-32.

Choi, A., \& Lam, E. (2016). Butterfly choices: Collecting stories of migrant sex workers around the world. Migrant Sex Workers'Justice Forum, 12 May, Ryerson University, Toronto, Ontario.

Durisin, E.M., Meulen, E.v.d., \& Bruckert, C. 2018. Contextualizing Sex Work: Challenging Discourses and Confronting Narratives. In Durisin, E.M., Meulen, E.v.d., \& Bruckert, C. (Eds.) Red Light Labour: Sex Work Regulation, Agency, and Resistance (pp. 3 - 27). Vancouver/Toronto: UBC Press.

Gallant, C., Lam, E., Zen, K., \& Chak, T. (2018). Migrant Sex Work Justice: A New Approach to the Anti-Trafficking Movement. In Haritaworn, J., Moussa, G., \& Ware, S.M. (Eds.) Marvelous Grounds: Queer of Colour Formations in Toronto (pp. 97 - 105). Toronto: Between the Lines.

Lam, E. (2016). The Birth of Butterfly: Bringing Migrant Sex Workers' Voices into the Justice for Sex Workers Movement. Research for Sex Work, (15), 6-7.

Lam, E. (2018a). Behind the Rescue: How Anti-trafficking Investigation and Policies Harm Migrant Sex Workers. Butterfly, Toronto, Canada. Retrieved from https://576a91ec-4a76-459b-8d054ebbf42a0a7e.filesusr.com/ugd/5bd754_bbd71c02 35c740e3a7d444956d95236b.pdf

Lam, E. (2018b). Survey on Toronto Holistic Practitioners' Experiences with Bylaw Enforcement and Police, Butterfly, Toronto, Canada. Retrieved from https://576a91ec-4a76-459b-8d054ebbf42a0a7e.filesusr.com/

Lam, E. (2019). How to develop a social justice framework to support migrant sex 30 workers and prevent the harm caused by anti-trafficking initiatives. Social Dialogue, (20), 30-34.

Lam, E. \& Gallant, C. (2018). Migrant sex work justice: building alliance across movement. In Durisin, E.M., Meulen, E.v.d., \& Bruckert, C. (Eds.). Red Light Labour: Sex Work Regulation, Agency, and Resistance (pp. 293 - 304). UBC Press.

Lam, E., \& Lepp, A. (2019). Butterfly: Resisting the harms of anti-trafficking policies and fostering peer-based organizing in Canada. Anti-Trafficking Review, (12), 91-107.

Lepp, A. (2018). Canada. In Global Alliance Against Traffic in Women (Ed.). Sex workers organizing for change: Self-representation, community mobilization, and working condition. (pp. 151-195). Retrieve from: https://www.gaatw. org/publications/SWorganising/SWorganisingcomplete-web.pdf

\section{Author Biographies}

Alvis Choi a.k.a. Alvis Parsley, born and raised in Hong Kong, is a nonbinary queer person whose artistic and curatorial traces can be seen in Toronto, Montreal, Faroe Islands, Berlin, Cardiff, Seoul, and Hong Kong. Alvis holds a Master of Environmental Studies with an area of concentration in Performance as Pedagogy and Community Transformation. Alvis has presented at the National Women's Studies Association, Performance Studies international (PSi) in Faroe Islands, and at the Critical Ethnic Studies Association Conference. From 2015-2017, Alvis was appointed as the Chairperson of the Chinese 
Canadian National Council Toronto Chapter. They are a collective member of Marvellous Grounds and have been involved with the work of Butterfly (Asian and Migrant Sex Workers Support Network) since 2015.

Elene Lam is an activist, artist, community organizer, educator, and human rights defender. She holds a Master of Social Work and a Master of Law, with a specialization in human rights. She is currently a PhD Candidate at McMaster University, where she is researching the harms associated with anti-trafficking initiatives. Elene has been actively engaged in work related to human rights, violence against women, migration, gender, and sex work justice for over 20 years. Elene is the Executive Director and Founder of Butterfly (Asian and Migrant Sex Workers Support Network). 